\title{
The Preference of Tryptophan for Membrane Interfaces ${ }^{\dagger}$
}

\author{
Wai-Ming Yau, ${ }^{\ddagger}$ William C. Wimley, ${ }^{\S}, l$ Klaus Gawrisch, ${ }^{\ddagger}$ and Stephen H. White*,§ \\ National Institute on Alcohol Abuse and Alcoholism, National Institutes of Health, 12420 Parklawn Drive, \\ Rockville, Maryland 20852, and Department of Physiology and Biophysics, University of California, \\ Irvine, California 92697-4560
}

Received April 10, 1998; Revised Manuscript Received June 25, 1998

\begin{abstract}
One of the ubiquitous features of membrane proteins is the preference of tryptophan and tyrosine residues for membrane surfaces that presumably arises from enhanced stability due to distinct interfacial interactions. The physical basis for this preference is widely believed to arise from amphipathic interactions related to imino group hydrogen bonding and/or dipole interactions. We have examined these and other possibilities for tryptophan's interfacial preference by using ${ }^{1} \mathrm{H}$ magic angle spinning (MAS) chemical shift measurements, two-dimensional (2D) nuclear Overhauser effect spectroscopy (2D-NOESY) ${ }^{1} \mathrm{H}$ MAS NMR, and solid state ${ }^{2} \mathrm{H}$ NMR to study the interactions of four tryptophan analogues with phosphatidylcholine membranes. We find that the analogues reside in the vicinity of the glycerol group where they all cause similar modest changes in acyl chain organization and that hydrocarbon penetration was not increased by reduction of hydrogen bonding or electric dipole interaction ability. These observations rule out simple amphipathic or dipolar interactions as the physical basis for the interfacial preference. More likely, the preference is dominated by tryptophan's flat rigid shape that limits access to the hydrocarbon core and its $\pi$ electronic structure and associated quadrupolar moment (aromaticity) that favor residing in the electrostatically complex interface environment.
\end{abstract}

A remarkable feature shared by the 10 or so membrane proteins (1) with known three-dimensional structures is the preference of $\operatorname{Trp}^{1}$ and Tyr residues for regions corresponding to membrane surfaces. A particularly striking example is illustrated in Figure 1. Statistical studies of sequence databases indicate that this preference is shared by virtually all membrane proteins $(2-4)$. Physicochemical studies of small peptides and Trp analogues suggest that the preference arises from enhanced stability due to distinct interfacial interactions $(5-10)$. This enhancement is commonly believed to originate from amphipathic interactions related to imino group hydrogen bonding (11), but specific dipolar interactions (6), enhanced acyl chain van der Waals interactions $(6,12)$, and cation $-\pi$ interactions $(13)$ have also been suggested. Interface-located Trp residues are important for gramicidin channel activity $(14-16)$, and aromatic residues have been implicated in the cation selectivity of potassium channels $(17,18)$ and the binding of ACh to ACh receptors (13). The fact that $\operatorname{Trp}$ has a higher propensity for the extracellular face of membrane proteins (2) (see Figure 1)

\footnotetext{
$\doteqdot$ This work was supported in part by Grant GM46823 to S.H.W from the National Institute of General Medical Sciences.

* To whom correspondence should be addressed. E-mail: shwhite@ uci.edu. Phone: (949) 824-7122. Fax: (949) 824-8540.

$\doteqdot$ National Institutes of Health.

$\S$ University of California.

"Present address: Department of Biochemistry SL-43, Tulane University School of Medicine, New Orleans, LA 70112-2699.

${ }^{1}$ Abbreviations: NMI, $N$-methylindole; $3 \mathrm{MI}, 3$-methylindole; POPC, 1-palmitoyl-2-oleoyl-sn-glycero-3-phosphocholine; POPC- $d_{31}$, 1-perdeuteriopalmitoyl-2-oleoyl-sn-glycero-3-phosphocholine; MAS, magic angle spinning; COSY, correlation spectroscopy; 2D-NOESY, twodimensional nuclear Overhauser effect spectroscopy; ACh, acetylcholine. Standard abbreviations are used for amino acids.
}

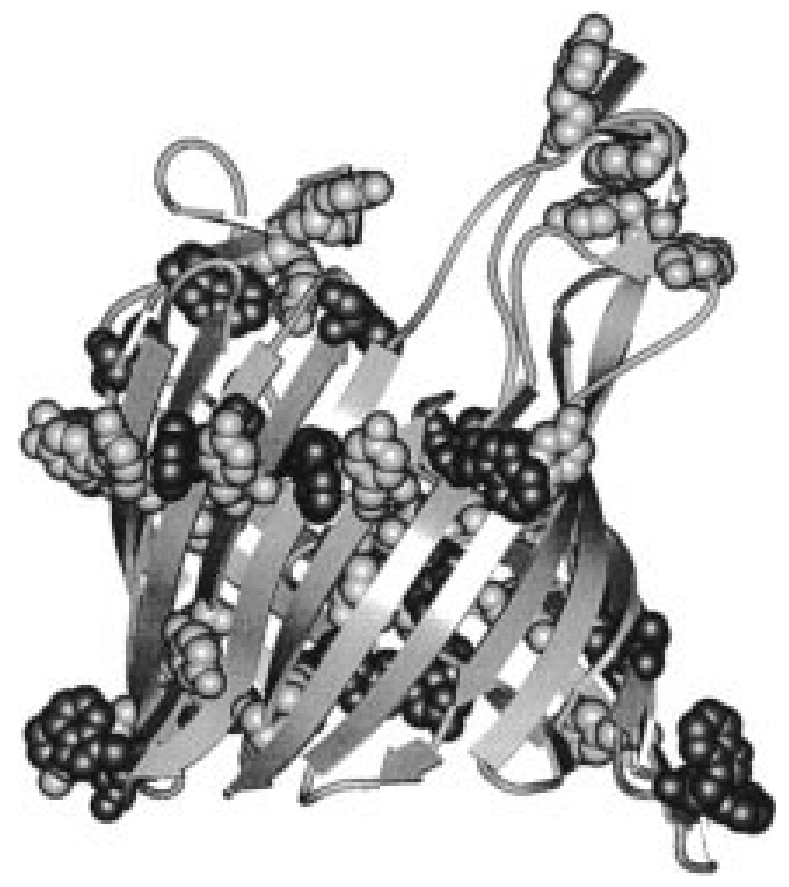

FIGURE 1: Structure of the membrane protein maltoporin showing the locations of all Trp and Tyr residues. The strands of the $\beta$-barrel backbone are shown as arrows, Trp residues black, and Tyr residues gray. The extracellular surface of the protein is at the top. Note that Tyr and Trp are particularly prominent on the extracellular surface. The image was constructed from the crystallographic coordinates $2 \mathrm{MPR}$ of maltoporin from Salmonella typhimurium determined by Meyer et al. (31).

suggests an important role in folding and assembly (11). The exact nature of the interactions of aromatic residues at the interface zones of membrane proteins is thus important to understand. 


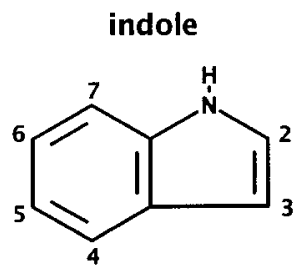

3-methylindole (3MI)

N-methylindole (NMI)
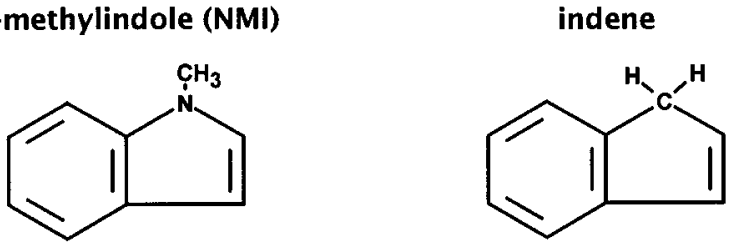

FIGURE 2: Chemical structures of the four Trp analogues used in this study.

As a first step toward clarifying the interfacial interactions of Trp, we studied the interactions of four carefully chosen Trp analogues with POPC membranes using ${ }^{1} \mathrm{H}$ MAS chemical shift measurements, 2D-NOESY ${ }^{1} \mathrm{H}$ MAS NMR, and solid state ${ }^{2} \mathrm{H}$ NMR. We were particularly interested in testing the hypotheses that imino group hydrogen bonding and/or dipole-dipole interactions might be the principal determinants of the interfacial preference. The four Trp analogues chosen (Figure 2) have similar water-membrane partition coefficients [indole, NMI, and 3MI, (6); indene (W. C. Wimley and S. H. White, unpublished)]. Indole, NMI, and 3MI have similar large dipole moments of about $2.1 \mathrm{D}$, whereas the dipole moment of indene is $0.67 \mathrm{D}(19)$. NMI and indene lack the ability to form imino group hydrogen bonds. We found that all of the analogues reside in the vicinity of the glycerol group and that hydrocarbon penetration was not increased by reduction of hydrogen bonding or dipole interaction potential, indicating that hydrogen bonding and dipole-dipole interactions are unlikely to be the primary determinants of Trp's interfacial preference. Rather, the dominant cause may arise from tryptophan's aromaticity (i.e., its $\pi$ electron structure and associated electrical quadrupole moment) that favors residing in the electrostatically complex interface environment and its flat rigid shape that limits access to the hydrocarbon core.

\section{MATERIALS AND METHODS}

Sample Preparation. POPC and POPC- $d_{31}$, obtained from Avanti Polar Lipids (Alabaster, AL), were $>98 \%$ pure as judged by chromatographic methods. Indole, 3-methylindole, $N$-methylindole, and indene obtained from Sigma (St. Louis, MO) were found to be $99.5 \%$ pure using high-pressure liquid chromatography. Approximately $10 \mathrm{mg}$ of POPC and the appropriate amount of the Trp analogue were dissolved in $1 \mathrm{~mL}$ of methanol. The solvent was then removed in a stream of argon. The lipid film was redissolved in $1 \mathrm{~mL}$ of cyclohexane which was removed by freeze-drying in a vacuum. The duration of the freeze-drying procedure was adjusted to remove the solvents but to maintain acceptable low losses of the volatile Trp analogues. The white Trp analogue/lipid powder was mixed with $20 \mu \mathrm{L}$ of $\mathrm{D}_{2} \mathrm{O}$ and homogenized by vortexing. This procedure results in large multilamellar liposomes that are in the liquid-crystalline lamellar phase $\left(\mathrm{L}_{\alpha}\right)$ at temperatures of $\geq 0{ }^{\circ} \mathrm{C}$. ${ }^{1} \mathrm{H}$ MAS
NMR spectra were used to determine the Trp analogue/POPC molar ratio and to check for residual solvent signals. Spectra were examined over an analogue concentration range from 0.1 to 1.8 per lipid. The Trp analogue-induced chemical shifts in the lipid spectra changed linearly with analogue concentration up to $1.0 \mathrm{Trp}$ analogue/lipid, indicating a homogeneous incorporation of molecules into the bilayers below this concentration. The partition coefficients of the analogues are independent of concentration near 0.5 analogue per lipid (6).

NMR Measurements. ${ }^{1} \mathrm{H}$ MAS NMR experiments were performed at $500.13 \mathrm{MHz}$ on a Bruker DMX500 wide-bore spectrometer at $10{ }^{\circ} \mathrm{C}$. Samples were spun at $5 \mathrm{kHz}$ using a Bruker double gas bearing MAS probe head for $4 \mathrm{~mm}$ rotors equipped with a deuterium lock. Lipid dispersions were kept inside a spherical $8 \mu \mathrm{L}$ volume of a Kel-F rotor insert. The ${ }^{1} \mathrm{H}$ chemical shift assignments of POPC and Trp analogue protons were confirmed by 2D MAS correlated NMR spectroscopy (COSY). 2D-NOESY MAS spectra were recorded in the phase sensitive mode as described previously (20). Cross-peak and diagonal peak intensities of indole and 3-methylindole were recorded as a function of mixing time ranging from 5 to $1000 \mathrm{~ms}$. Peak volumes were calculated with Bruker AURELIA software. The data were fitted to a spin pair interaction model (21). Experiments at a single mixing time of $300 \mathrm{~ms}$ were conducted on all Trp analogue/POPC samples. Approximate cross-relaxation rates, $\sigma_{\mathrm{BA}}$, were calculated with the approximate formula $\sigma_{\mathrm{BA}}$ $=I_{\mathrm{BA}} /\left(I_{\mathrm{AA}} \tau\right)$, where $I_{\mathrm{BA}}$ is the cross-peak volume, $I_{\mathrm{AA}}$ the lipid diagonal peak volume from which migration of magnetization starts, and $\tau$ the mixing time. The single mixing time analysis is a suitable alternative when relative rate differences between structurally close lipid systems are compared. The mixing time of $300 \mathrm{~ms}$ was chosen for the optimal cross-peak intensity with minimal distortions from spin-lattice relaxation effects. NMR experiments on protonated DMPC in a perdeuterated DMPC- $d_{67}$ matrix have shown that NOESY cross-relaxation rates in lipid bilayers are almost exclusively of intermolecular origin. The data rule out magnetization transfer by spin diffusion between distant protons within lipid molecules (D. Huster, K. Arnold, and $\mathrm{K}$. Gawrisch, submitted for publication).

Solid state ${ }^{2} \mathrm{H}$ NMR measurements were conducted at 46.07 MHz on a Bruker DMX300 spectrometer (Billerica, MA) with a quadrupolar echo sequence (22) using a $2.2 \mathrm{~ms}$ $90^{\circ}$ excitation pulse, a $50 \mu$ s delay between pulses, and a relaxation delay of $500 \mathrm{~ms}$. Typically, 8192 scans were accumulated at $25^{\circ} \mathrm{C}$ using a spectral width of $200 \mathrm{kHz}$. Smoothed order parameter profiles of the perdeuterated palmitoyl chain were calculated according to the procedure of Lafleur et al. (23).

\section{RESULTS AND DISCUSSION}

The results of NMR measurements reported here were realized with fully hydrated liquid-crystalline POPC bilayers containing the analogues at concentrations of about 0.7 per lipid. Figure 3 a shows the structure of POPC and identifies the specific groups whose interactions with the Trp analogues could be determined. Aromatic ring current-induced changes in lipid proton chemical shifts (Figure 4) and NOESY crossrelaxation rates between lipid and Trp analogue proton 

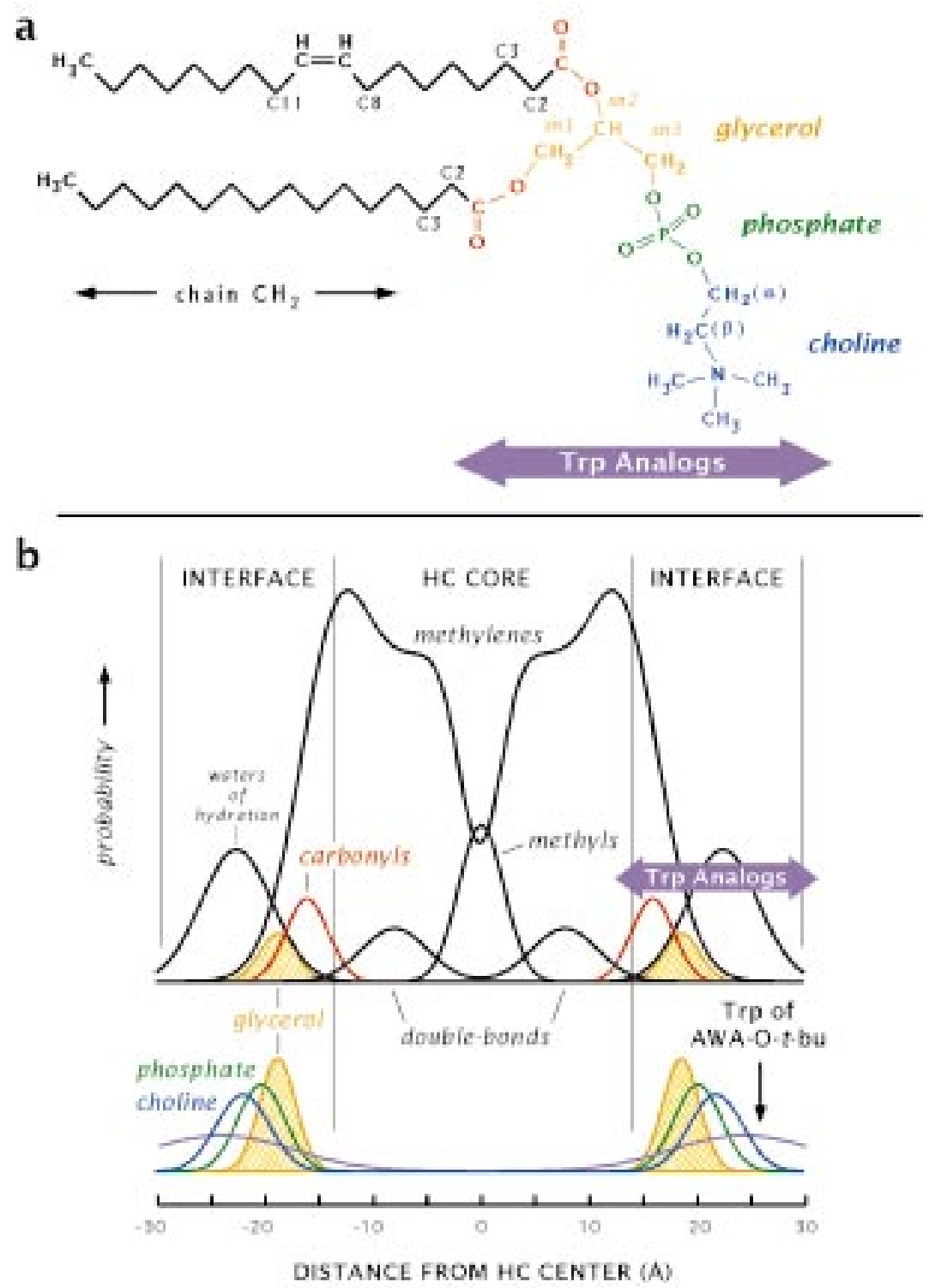

FIGURE 3: Chemical structure of palmitoyloleoylphosphatidylcholine (POPC) and liquid-crystallographic structure of dioleoylphosphatidylcholine (DOPC). (a) Chemical structure of a POPC molecule showing the locations of the protons that were individually observed in the ${ }^{1} \mathrm{H}$ MAS NMR spectroscopy experiments. The lipid region with the strongest interactions with the Trp analogues is shown by the broad arrow. (b) Structure of a fluid-phase dioleoylphosphatidylcholine (DOPC) bilayer. This fully resolved structural image (25), consisting of the time-averaged transbilayer distributions of the principal lipid structural groups and water, is obtained by joint liquidcrystallographic refinement of X-ray and neutron diffraction data from fluid ( $\mathrm{L}_{\alpha}$-phase) lipid bilayers (32, 33). The distributions are Gaussian number or probability densities that represent time-averaged projections of the three-dimensional motions of the groups onto the bilayer normal and give the probability of finding a structural group at a particular transbilayer location. The area under each distribution is the number of groups in the lipid (one glycerol, two carbonyls, etc.). The methylene distribution is the sum of three Gaussian distributions, and the water distribution is the water of hydration of the headgroup. Also shown is the distribution of the Trp residue of Ala-Trp-Ala-O-tertbutyl as determined by neutron diffraction (5). Notice that the Trp spends little time in the hydrocarbon core of the bilayer; it is located in the same region as the water of hydration of the headgroup.

resonances (Figure 5) were used to determine the location of the analogues. Solid state ${ }^{2} \mathrm{H}$ NMR measurements of lipid acyl chain order parameters were used to assess the analogueinduced order changes in the membranes.

The aromatic ring current-induced chemical shifts of lipid protons indicate an interfacial location of the four compounds (Figure 6a). These shifts, arising from protons that are close to the aromatic rings, can be either upfield or downfield, depending on the proton's location relative to the plane of the ring. All lipid shifts were upfield, indicating that, on average, lipid protons are located above the aromatic ring systems (24). This is consistent with ${ }^{2} \mathrm{H}$ NMR experiments on deuterated Trp analogues in bilayers which indicate that the indole ring has a preferential orientation parallel to the bilayer normal while maintaining a high level of motional freedom (W.-M. Yau and K. Gawrisch, unpublished). Proton resonances of the upper acyl chain segments near the glycerol showed the largest induced shifts. Indole and 3MI also shifted lipid choline resonances to a significant degree, but headgroup proton shifts were seen only at high Trp analogue concentrations. At these high concentrations, the more water-soluble indole and 3MI have increased probabilities of being located in the water phase near the headgroups. The 2D-NOESY cross-relaxation rates between the analogues and lipid proton resonances (Figure $6 b$ ) reveal their close throughspace proximity. They show that protons of all four 


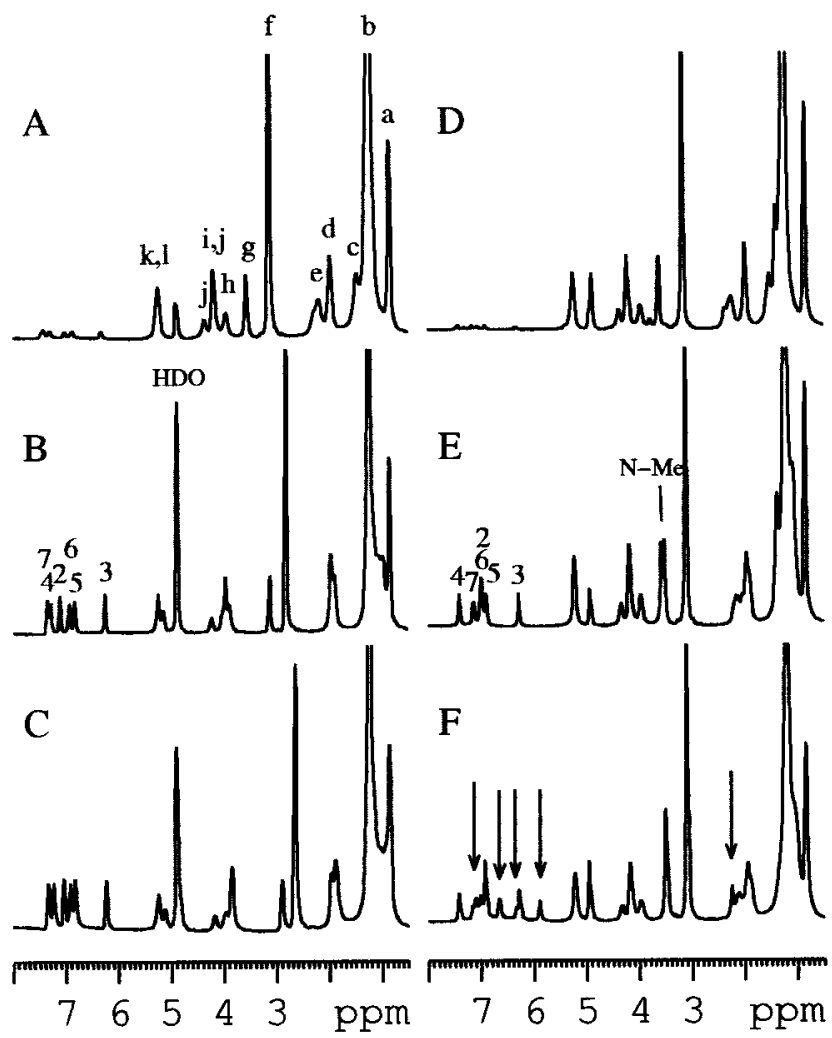

FIGURE 4: ${ }^{1} \mathrm{H}$ MAS NMR spectra of $\mathrm{POPC} / \mathrm{D}_{2} \mathrm{O}$ dispersions containing variable amounts of indole or $N$-methylindole (NMI). For panels $\mathrm{A}-\mathrm{C}$, molar ratios of indole/POPC are $0.17,1.09$, and 1.72 , respectively. For panels $\mathrm{D}-\mathrm{F}$, ratios of NMI/POPC are 0.08 , 1.09 , and 1.70, respectively. Incorporation of Trp analogues into POPC bilayers shifts lipid resonances of the lipid-water interface to higher fields. The solubility of NMI in POPC is limited to molar ratios below 1. At higher concentrations, the excess NMI forms oily droplets that separate from the lipid. The resonance signals of the separated NMI are highlighted with arrows (panel F). Lipid resonance assignments indicated in panel $\mathrm{A}$ are as follows: (a) chain $\mathrm{CH}_{3}$, (b) chain $\mathrm{CH}_{2}$, (c) $\mathrm{C} 3$, (d) $\mathrm{C} 8$ and $\mathrm{C} 11$, (e) $\mathrm{C} 2$, (f) $\mathrm{N}\left(\mathrm{CH}_{3}\right)_{3}$, (g) $\mathrm{CH}_{2}(\beta)$, (h) $s n-3$, (i) $\mathrm{CH}_{2}(\alpha)$, (j) $s n-1$, (k) $s n-2$, and (l) $\mathrm{CH}$. Panels $\mathrm{B}$ and $\mathrm{E}$ show the Trp analogue assignments.

analogues have fast cross-relaxation rates with the glycerol protons and the upper acyl chain protons, but much slower rates with the protons of the hydrocarbon core or the phosphocholine groups. Both the ring current-induced chemical shifts of POPC resonances and NOESY crossrelaxation rates are consistent with a preferential location of all four of the chemically distinct analogues in the membrane interface. Although there are subtle differences among them, the data indicate that differences in hydrogen bonding and electric dipole interaction ability have little effect on the transmembrane positions of the analogues.

Solid state ${ }^{2} \mathrm{H}$ NMR orientational order parameter profiles, obtained through perdeuteration of the palmitoyl chain of POPC (POPC- $d_{31}$ ), allow the effects of the Trp analogues on the structure of the hydrocarbon core to be assessed so the possibility of enhanced acyl chain van der Waals interactions can be examined $(6,12)$. Figure 7 shows that all four analogues slightly increase POPC- $d_{31}$ chain order near the interface and decrease chain order in the bilayer core. These changes are consistent with an interfacial location of the analogues that leads to ordering of upper chain segments by direct analogue-chain contacts and disordering of lower chain segments due to the accompanying increase

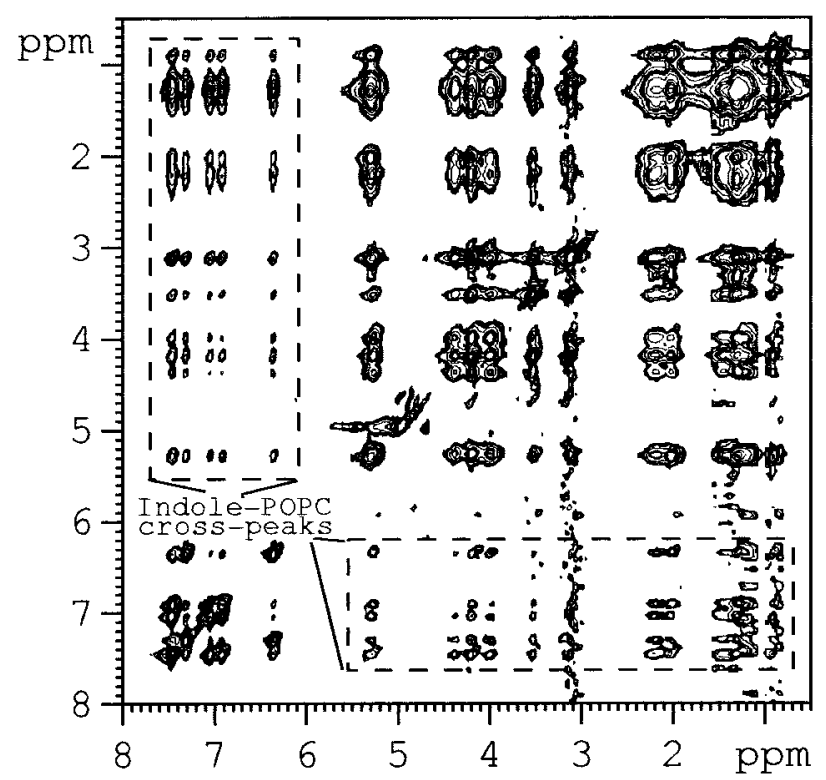

FIGURE 5: Two-dimensional ${ }^{1} \mathrm{H}$ MAS NMR NOESY spectrum of an indole/POPC $(0.41 \mathrm{~mol} / \mathrm{mol})$ dispersion in $\mathrm{D}_{2} \mathrm{O}$ recorded with a mixing time of $300 \mathrm{~ms}$ and at a temperature of $10^{\circ} \mathrm{C}$. The spectrum shows intense cross-peaks between indole and POPC resonances.

in lateral chain area. Overall, there is no net ordering of the acyl chains of the sort necessary for enhanced acyl chain van der Waals interactions because the large enthalpies observed for the analogue-bilayer interactions (see below) are equivalent to the liquid-to-gel solidification of 10-14 mol of $\mathrm{CH}_{2}$ per mole of indole (6). We conclude that increased acyl chain packing density cannot be the basis for the favorable interfacial interactions of our Trp analogues.

A structural context for these NMR results is provided by Figure $3 b$ which shows the structure of a fluid-phase dioleoylphosphocholine (DOPC) bilayer membrane (25) determined by joint refinement of X-ray and neutron diffraction data. The bilayer structure consists of the collection of the transbilayer distributions of structural groups comprising the lipid. Each distribution represents the time-averaged projection of a group's three-dimensional motion onto the bilayer normal. One of the salient features of the image is the large thermal disorder that gives rise to a broad, dynamic, and chemically heterogeneous interfacial region composed of the headgroups, water, and portions of acyl chain methylenes that spill into the interface from the hydrocarbon core. This means that there is no distinct boundary between the upper acyl chain, glycerol, and headgroup regions. In the context of this image, the induced chemical shifts (Figure 6a) and the distribution of NOE cross-relaxation rates (Figure 6b) place the Trp analogues in this region of tumultuous chemical heterogeneity. Remarkably, the indole ring of the tripeptide Ala-Trp-Ala-O-tert-butyl has a transbilayer distribution in fluid DOPC bilayers (Figure 3b) that is similar to that of the analogues despite the indole ring's covalent linkage to the tripeptide. This distribution, determined directly using neutron diffraction (5), shows that the indole ring spends most of its time in the vicinity of the glycerophosphocholine group and water with little penetration into the hydrocarbon core. Collectively, these data show clearly that Trp and its analogues are preferentially located within the bilayer interface with little penetration into the hydrocarbon core (Figure 3). This lack of penetration is unex- 

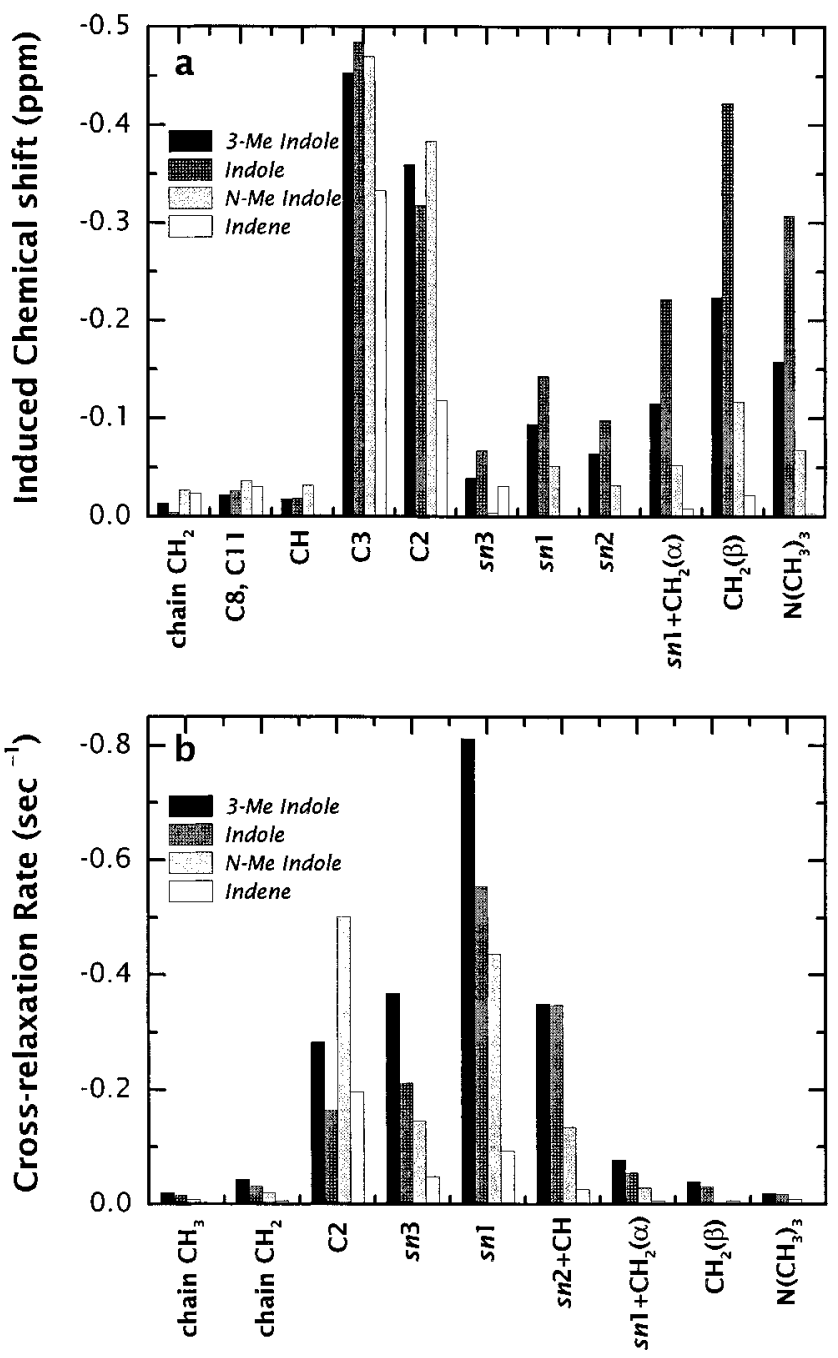

FIGURE 6: Aromatic ring current-induced chemical shifts of POPC protons and rates of NOE cross-relaxation between lipid and Trp analogue protons. (a) Trp analogue-induced changes in POPC proton chemical shifts. Pure POPC chemical shifts were subtracted from the shifts in the presence of the analogues. The Trp analogue/ POPC mole fractions are 0.64 for $3 \mathrm{MI}, 0.64$ for indole, 0.76 for $\mathrm{NMI}$, and 0.79 for indene. In the presence of Trp analogues, the chemical shifts of C2 and C3 methylene resonances of $s n-1$ and $s n-2$ chains are nonequivalent and shift upfield at slightly different rates. Only the shifts with the larger magnitude are shown. (b) Rates of cross-relaxation between POPC and indole, 3-methylindole, $\mathrm{N}$-methylindole, and indene protons in large multilamellar POPC liposomes. The Trp analogue/POPC molar ratios are 0.64 for $3 \mathrm{MI}$, 1.09 for indole, 0.76 for NMI, and 0.79 for indene. Cross-relaxations from lipid resonances to the following Trp analogue resonances are shown: 3-methyl of 3MI, 3-H of indole, 3-H of NMI, and 3-H of indene. All chosen resonances originate from very similar locations on the pyrrole ring. Rates of cross-relaxation between the lipid and other indole resonances are similar. For NMI and indene, the $\mathrm{OCH}_{2} \mathrm{CH}(s n-2)$ and vinyl proton cross-relaxation rates are superimposed. The rates of cross-relaxation between choline methyl and indene methylene resonances, and between $\beta$-choline and NMI methyl resonances, could not be determined because of signal superposition.

pected because the analogues all partition strongly into bulk hydrocarbon phases from water (26). Perhaps the rigid disklike shape cannot be accommodated without unfavorable entropic consequences due to ordering of the bilayer acyl chains. In any case, Trp tends to be excluded from the bilayer interior.

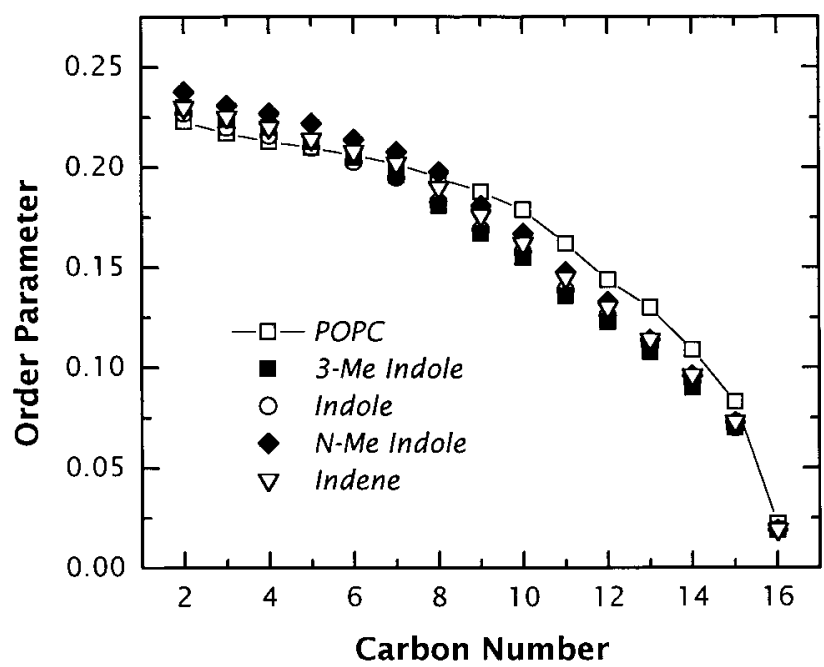

FIGURE 7: Solid state ${ }^{2} \mathrm{H}$ order parameters along the deuterated palmitoyl chain of POPC in the absence and presence of the four Trp analogues at $25^{\circ} \mathrm{C}$. The Trp analogue/POPC molar ratios are 0.54 for $3 \mathrm{MI}, 0.57$ for indole, 0.89 for NMI, and 0.71 for indene. The assignment of order parameters was made assuming a continuous order decrease from the interface to the bilayer center.

The similarities of the interfacial locations of the four Trp analogues determined in our NMR experiments indicate that neither imino group hydrogen bonding nor electric dipolar interactions are solely responsible for the interfacial localization because NMI lacks the hydrogen bonding ability of indole and 3MI, and indene's ability to participate in either hydrogen bond or dipole interactions is greatly reduced. Although choline cation $-\pi$ interactions may play a role in the interfacial interactions, they do not appear to be of primary importance because the lipid proton chemical shifts in the choline region vary greatly among the analogues (Figure 6a) and the strengths of choline NOE cross-relaxation rates are small in comparison to those associated with the glycerol and upper chain regions (Figure 6b). Our results thus indicate that specific hydrogen bonding, dipole, and cation $-\pi$ interactions cannot individually explain the interfacial preference of Trp and its analogues, although all of them no doubt contribute to the exact placement and orientation.

Are there other possibilities for tryptophan's interfacial preference? We suggest that interactions of a more general nature involving aromaticity and molecular shape play a dominant role. Given the fact that the hydrophobic effect acts to exclude the Trp analogues from water, as revealed clearly by water-cyclohexane partitioning measurements $(26,27)$, the issue is whether the preferred location of the water-excluded analogues is the hydrocarbon core or the bilayer interfaces (see Figure $3 b$ ). We suggested above that the flat, rigid shape of the Trp analogues may cause them to be excluded from the hydrocarbon core for entropic reasons. Although the details of the exclusion process remain to be determined, the hydrocarbon core apparently behaves as a strongly "cohesive" material that disfavors the deep insertion of Trp analogues. At the same time, electrical characteristics peculiar to aromatic molecules $(13,28)$ may make the analogues ideally suited to residing in the complex electrostatic environment of the hydrated glycerophosphocholine groups. 
The defining electrical characteristic of aromatic molecules is an electrical quadrupole moment arising from their $\pi$ electrons that may be roughly visualized as two "tail-to-tail" dipoles arranged with their positively charged tails centered on the midplane of the aromatic ring (see ref 13). This quadrupole moment is primarily responsible for the cation $-\pi$ interaction. Secondarily, aromatic molecules with asymmetric ring systems can also have dipole moments because of asymmetries in electron density distribution over the ring system. Such is the case for the Trp analogues. But, quadrupolar and dipolar interactions alone are not sufficient for understanding the energetics of interactions of the Trp analogue with the headgroup; additional terms such as induced dipoles, polarizabilities, dispersion forces, and charge transfer must also be included (29). We suggest that these properties of the Trp analogues, referred to collectively as aromaticity, give rise to favorable interactions with the complex charge distribution in the interface region of the bilayer.

The idea that aromaticity is important is supported by simple thermodynamic measurements $(6,26,27)$. The free energies of transfer of indole, 3MI, and NMI into cyclohexane from water are very favorable $(-4.4$ to $-7.2 \mathrm{kcal} / \mathrm{mol})$ and are dominated by entropy due to the hydrophobic effect. The relatively small enthalpies $(1.1-2.6 \mathrm{kcal} / \mathrm{mol})$ associated with the transfer are unfavorable as expected from the observation that aromatic compounds can form hydrogen bonds with water $(13,30)$. The enthalpic cost for indole and $3 \mathrm{MI}$ is $1.2 \mathrm{kcal} / \mathrm{mol}$ higher than that for NMI because of the hydrogen bonding capacity of the imino group. In contrast, the free energies of transfer into phosphatidylcholine bilayers are about the same as that for cyclohexane $(-7.6$ to $-8.6 \mathrm{kcal} / \mathrm{mol})$, but are composed of very favorable enthalpies $(-3.4$ to $-5.4 \mathrm{kcal} / \mathrm{mol})$ as well as favorable entropies due to the hydrophobic effect (6). The negative enthalpies are consistent with favorable interactions arising from the presence of the indole compounds in the bilayer interface.

The interfacial location of tryptophan may therefore simply be due to the balance of the hydrophobic effect that tends to drive it out of water, complex electrostatic interactions that favor residing in the hydrated headgroup region, and cohesive repulsion that keeps it out of the hydrocarbon core. In this scenario, dipolar, quadrupolar, $\mathrm{H}$ bonding, and cation $-\pi$ interactions act together to determine the precise disposition of Trp in the interface. The differences in the NMR data for the Trp analogues seen in Figure 6, indicative of subtle changes in analogue disposition, probably represent variations in the relative contributions of these various interactions.

\section{ACKNOWLEDGMENT}

We thank Dr. Dennis Dougherty for useful conversations about cation $-\pi$ interactions.

\section{REFERENCES}

1. Preusch, P. C., Norvell, J. C., Cassatt, J. C., and Cassman, M. (1998) Nat. Struct. Biol. 5, 12-14.

2. Landolt-Marticorena, C., Williams, K. A., Deber, C. M., and Reithmeier, R. A. F. (1993) J. Mol. Biol. 229, 602-608.

3. von Heijne, G. (1994) Annu. Rev. Biophys. Biomol. Struct. 23, 167-192.
4. Reithmeier, R. A. F. (1995) Curr. Opin. Struct. Biol. 5, 491500 .

5. Jacobs, R. E., and White, S. H. (1989) Biochemistry 28, 34213437.

6. Wimley, W. C., and White, S. H. (1993) Biochemistry 32, 6307-6312.

7. Kachel, K., Asuncion-Punzalan, E., and London, E. (1995) Biochemistry 34, 15475-15479.

8. Wimley, W. C., and White, S. H. (1996) Nat. Struct. Biol. 3, $842-848$.

9. Killian, J. A., Salemink, I., de Planque, M. R. R., Lindblom, G., Koeppe, R. E., II, and Greathouse, D. V. (1996) Biochemistry 35, 1037-1045.

10. Morein, S., Strandberg, E., Killian, J. A., Persson, S., Arvidson, G., Koeppe, R. E., II, and Lindblom, G. (1997) Biophys. J. 73, 3078-3088.

11. Schiffer, M., Chang, C. H., and Stevens, F. J. (1992) Protein Eng. 5, 213-214.

12. Beschiaschvili, G., and Seelig, J. (1992) Biochemistry 31, 10044-10053.

13. Dougherty, D. A. (1996) Science 271, 163-168.

14. Ketchem, R. R., Hu, W., and Cross, T. A. (1993) Science 261, $1457-1460$.

15. Hu, W., Lee, K. C., and Cross, T. A. (1993) Biochemistry 32, 7035-7047.

16. Koeppe, R. E., Killian, J. A., and Greathouse, D. V. (1994) Biophys. J. 66, 14-24.

17. Durell, S. R., and Guy, H. R. (1992) Biophys. J. 62, 238250.

18. Bogusz, S., Boxer, A., and Busath, D. D. (1992) Protein Eng. $5,285-293$.

19. McClellan, A. L. (1963) in Tables of Experimental Dipole Moments, W. H. Freeman, San Francisco.

20. Holte, L. L., and Gawrisch, K. (1997) Biochemistry 36, 46694674.

21. Ernst, R. R., Bodenhausen, G., and Wokaun, A. (1990) in Principles of Nuclear Magnetic Resonance in One and Two Dimensions, Clarendon Press, New York.

22. Davis, J. H., Jeffrey, K. R., Bloom, M., Valic, M. I., and Higgs, T. P. (1976) Chem. Phys. Lett. 42, 390-394.

23. Lafleur, M., Fine, B., Sternin, E., Cullis, P. R., and Bloom, M. (1989) Biophys. J. 56, 1037-1041.

24. Stamm, H., and Jäckel, H. (1989) J. Am. Chem. Soc. 111, 6544-6550.

25. Wiener, M. C., and White, S. H. (1992) Biophys. J. 61, 434447.

26. Wimley, W. C., and White, S. H. (1992) Biochemistry 31, $12813-12818$

27. Wimley, W. C., and White, S. H. (1993) Biochemistry 32, 9262.

28. Mecozzi, S., West, A. P., Jr., and Dougherty, D. A. (1996) Proc. Natl. Acad. Sci. U.S.A. 93, 10566-10571.

29. Lee, S., Yoshitomi, H., Morikawa, M., Ando, S., Takiguchi, H., Inoue, T., and Sugihara, G. (1995) Biopolymers 36, 391398.

30. Suzuki, S., Green, P. G., Bumgarner, R. E., Dasgupta, S., Goddard, W. A., III, and Blake, G. A. (1992) Science 257, 942-945.

31. Meyer, J. E. W., Hofnung, M., and Schulz, G. E. (1997) J. Mol. Biol. 266, 761-775.

32. Wiener, M. C., and White, S. H. (1991) Biophys. J. 59, 162173.

33. Wiener, M. C., and White, S. H. (1991) Biophys. J. 59, $174-185$.

BI980809C 